\title{
Merging Directed C-H Activations with High-Throughput Experimentation: Development of Predictable Iridium-Catalyzed C-H Aminations Applicable to Late-Stage Functionalization
}

\author{
Erik Weis, ${ }^{a, b}$ Maria Johansson, ${ }^{c}$ Pernilla Korsgren, ${ }^{c}$ Belén Martín-Matute, ${ }^{* a}$ Magnus J. Johansson*a,b \\ ${ }^{a}$ Department of Organic Chemistry, Stockholm University, Stockholm SE-106 91, Sweden, \\ ${ }^{\mathrm{b}}$ Medicinal Chemistry, Research and Early Development; Cardiovascular, Renal and Metabolism, Biopharmaceuticals R\&D, \\ AstraZeneca,Gothenburg, Pepparedsleden 1, 43150 Mölndal, Sweden \\ ${ }^{c}$ Compound Synthesis and Management, Discovery Sciences, BioPharmaceuticals R\&D, AstraZeneca, Gothenburg, \\ Pepparedsleden 1, 43150 Mölndal, Sweden \\ * Correspondence to: belen.martin.matute@su.se (B.M.-M.) Magnus.J.Johansson2@astrazeneca.com (M.J.J.)
}

\begin{abstract}
Herein, we report an iridium-catalyzed directed $\mathrm{C}-\mathrm{H}$ amination methodology developed using a highthroughput experimentation (HTE)-based strategy, applicable for the needs of automated modern drug discovery. The informer library approach for investigating accessible directing group chemical space for the reaction, in combination with functional group tolerance screening and substrate scope investigations, allowed for the generation of an empirical predictive model to guide future users. Applicability to late-stage functionalization of complex drugs and natural products, in combination with multiple deprotection protocols leading to the desirable aniline matched pairs, serve to demonstrate the utility of the method for drug discovery. Finally reaction miniaturization to a nanomolar range highlights the opportunities for more sustainable screening with decreased material consumption.
\end{abstract}

\section{Introduction}

Innovation in synthetic organic chemistry is of fundamental importance to the improvement of the drug discovery process. While the field has seen tremendous developments over the past century, recent advances in synthetic methods, chemoinformatics, and increasing applicability of automation and miniaturization in synthesis have the potential to further transform and improve modern drug discovery. ${ }^{1-4}$ Two particular technological and synthetic approaches stand at the forefront of our interest and focus in this work: high-throughput experimentation (HTE), and $\mathrm{C}-\mathrm{H}$ functionalization. HTE techniques attracted significant interest from the pharmaceutical industry, and are now increasingly utilized in the drug discovery process. ${ }^{5}$ From the methodology development perspective the advantages are clear: access to more high-quality and well-rounded results with decreased material and time cost associated. Adding to this is the importance large, high quality datasets for the generation of predictive reactivity models. ${ }^{6,7}$ At the same time, reaction miniaturization allows for more sustainable chemistry by means of decreased material consumption, including reagents, solvents, and especially high-value advanced intermediates and catalysts. Finally, technologies such as automated liquid and solid dispensing allow chemists to avoid repetitive non-intellectual tasks, while providing high reproducibility and evading the risk for human error in setting up large arrays. Given the abundance of $\mathrm{C}-\mathrm{H}$ bonds in drugs and their building blocks, $\mathrm{C}-\mathrm{H}$ functionalizations are among the most desirable transformations in drug discovery. Of particular interest are late-stage functionalizations (LSF), ${ }^{8,9}$ where the controlled chemoselective transformation of desired $\mathrm{C}-\mathrm{H}$ bonds in complex drug-like molecules has the potential to greatly aid the hit-to-lead and lead-optimization processes. ${ }^{10}$ Bypassing the need for time, material and labor intensive de novo synthesis of analogues would greatly aid structure-activity relation (SAR) studies, or even the generation of new candidate drugs. In terms of desirable transformations, the introduction of small functional groups like $-\mathrm{CH}_{3}$, $\mathrm{CF}_{3},-\mathrm{NH}_{2},-\mathrm{OH}$ and $-\mathrm{F}$ are of highest priority, and would be widely used in the industry. ${ }^{1}$ Further motivating the development of new amination methodologies, a recent analysis of $\mathrm{X}$-ray structural data identified $\mathrm{N}-\mathrm{H}$ hydrogen bond donors on aromatic and aliphatic amines as the most common 
a) $\mathrm{C}\left(\mathrm{sp}^{2}\right)-\mathrm{NH}_{2}$ in pharmaceuticals<smiles>Nc1c(CC(=O)O)cccc1C(=O)c1ccc(Br)cc1</smiles><smiles>CCOCCOc1cc(-c2cnn(C3CCNCC3)c2)cnc1N</smiles><smiles>CCN(CC)CCNC(=O)c1ccc(N)cc1</smiles>

b) LSF: Challenges and opportunities
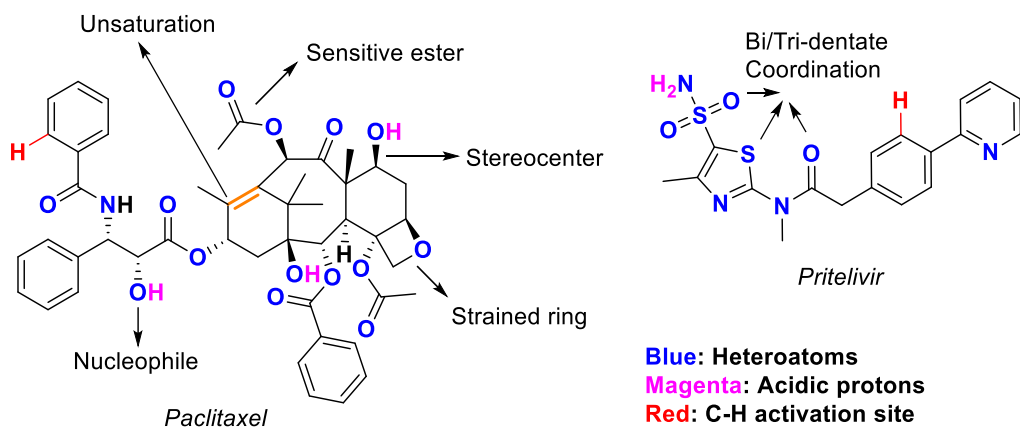

Figure 1. a) Selected examples of drugs containing an aniline moiety b) Representative examples of challenging functional groups encountered with LSF applications and opportunities for directed $\mathrm{C}-\mathrm{H}$ activations.

polar functional groups involved in fragment-protein binding (For examples of anilines see Figure 1, a). ${ }^{2}$ Directed $\mathrm{C}-\mathrm{H}$ activations offer a means of introducing amine moieties in the vicinity of Lewis basic groups commonly present in drug-like molecules with high regioselectivity. Over the past decade a number of methodologies for $\mathrm{C}\left(\mathrm{sp}^{2}\right)-\mathrm{H}$ to $\mathrm{C}-\mathrm{N}$ bond transformation have been developed, ${ }^{10-14}$ utilizing among others $\mathrm{Co},{ }^{15,}{ }^{16} \mathrm{Rh}^{17-19} \mathrm{Ir}^{20-24}$ and $\mathrm{Ru}^{25-28}$ catalysts. However, applicability for LSF in a drug discovery context remains challenging. We identified several factors which limit the utility of reported $\mathrm{C}-\mathrm{H}$ aminations in this respect. Firstly, the inaccessibility of free amines. The majority of reported directed $\mathrm{C}-\mathrm{H}$ to $\mathrm{C}-\mathrm{N}$ bond forming reactions, while introducing protected amines in form of amides and sulfonamides, do not include deprotection protocols. Although the introduction of larger substituents can be of utility for fragment-based drug discovery, applications to LSF are of limited use if the free amines cannot be obtained under mild-enough conditions to tolerate a large array of reactive and/or sensitive functional groups present in drug-like molecules. Secondly, the limited functional group tolerance. A common shortcoming of the reported procedures is a lack of compatibility with polar functional groups commonly present in drug-like molecules, such as heterocycles, alcohols, amines, carboxylic acids or amides (practical examples in Figure 1, b). The third limitation is closely related to this, and it is the lack of reporting of unsuccessful transformations and limited number of reports on applicability to complex substrates. This situation would be largely mitigated by full disclosure of the investigated substrate scope. Aside from this, two distinct approaches have been recently developed to improve the predictability of chemical methods in a more systematic fashion; the intermolecular robustness screening approach developed by the Glorius group, ${ }^{29-31}$ and the chemistry informer library approach by Krska and coworkers. ${ }^{7,32}$ The intermolecular robustness screening approach evaluates the compatibility of additives bearing a wide variety of functional groups with the transformation of a single substrate. In the informer library approach, the compatibility of a methodology with a large number of complex substrates bearing structural features relevant to pharmaceuticals is evaluated. While the former has been previously used for reported $\mathrm{C}-\mathrm{H}$ activation methodologies, ${ }^{33,34}$ the latter has so far only been applied to more well established crosscoupling reactions. ${ }^{7}, 32$

Herein we report the development of an iridium-catalyzed directed ortho-C-H amination applicable to a large number of directing groups (DGs) with outstanding functional group tolerance and 
regioselectivity. The use of $\left[\mathrm{Cp} * \operatorname{Ir}\left(\mathrm{H}_{2} \mathrm{O}\right)_{3}\right] \mathrm{SO}_{4}$ catalyst allows for regioselective functionalization governed by DGs inherently present in building blocks, drugs and natural products, without the need for additional ligands. An empirical predictive model based on a DG informer library, functional group tolerance studies and LSF informer library serves to guide potential users in predicting reaction applicability for complex substrates. The obtained Moz-protected amines can be deprotected under three distinct conditions, further increasing the utility of the amination protocol to complex molecules.

\section{Results and discussion}

At the beginning of the study we designed a workflow (Figure 2) which, if successful, would deliver reaction conditions for LSF applications. In the initial stage an optimization study to find suitable screening conditions was undertaken (Figure 2, Step 1).

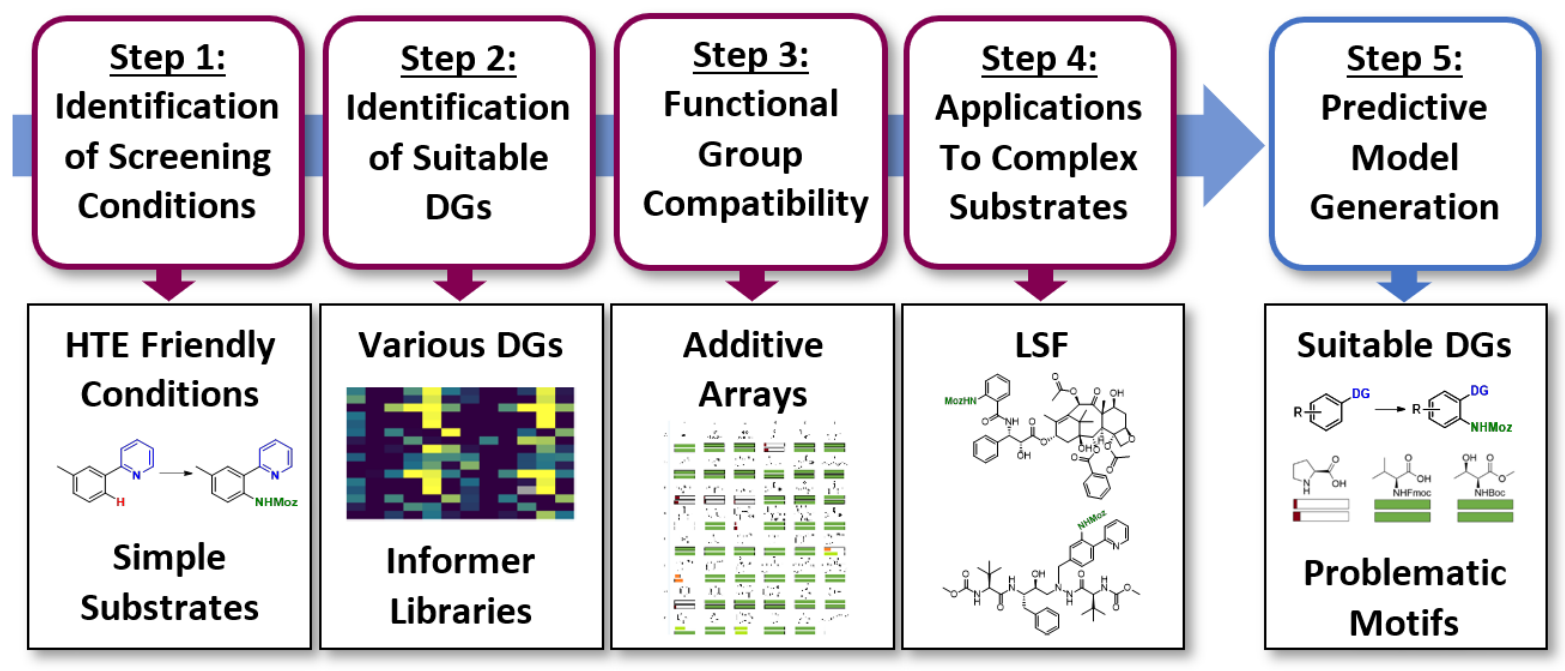

Figure 2. Strategy for HTE-enabled reaction discovery and applicability investigations for methodology development. DG = directing group.

In terms of reaction conditions, we identified several desirable features of "ideal" $\mathrm{C}-\mathrm{H}$ activation methodologies applicable to HTE. ${ }^{34-36}$ These include: 1) Use of soluble reagents and liquid dispensing. Beyond the ease of setting up complex libraries, this would also allow further decreasing the reaction scale with maintained reproducibility, surpassing the limitations of solid dispensing methods. 2) Reactions tolerant of moisture, allowing for direct use of reagents and large compound libraries without the need of rigorous drying. 3) Reactions tolerant to air atmosphere. 4) Commercially available reagents. 5) Compatibility with plastic reaction vessels. 6) Compatibility with non-volatile reaction solvents. The last 2 points are crucial for miniaturization and use of 384- and 1536-well reaction plate formats.

The following set of conditions was identified based on these criteria after Step 1, initial optimizations (see $\mathrm{SI}$ ), and used for the directing group informer library. $\left[\mathrm{Cp} * \operatorname{Ir}\left(\mathrm{H}_{2} \mathrm{O}\right)_{3} \mathrm{SO}_{4}\right]$ was chosen as catalyst, ${ }^{34}$, ${ }^{37}$ allowing the reaction to be performed in absence of silver salts and insoluble additives, thus facilitating the use of liquid handling systems. Commercially available $\mathrm{MozN}_{3}$ (Moz $=p$ methoxybenzyloxycarbonyl) was selected as the nitrogen source, allowing for deprotection of the obtained carbamate under a number of conditions. ${ }^{38,39}$ Although the transformation was performed with a satisfactory outcome in a wide range of solvents (see $\mathrm{SI}$ ), four were chosen for the informer library: 1,2-dichloroethane (DCE), which performed best in the initial study, cyclopentyl methyl ether (CPME) and EtOAc as greener solvent alternatives, and $\mathrm{N}$-methyl-2-pyrrolidone (NMP) for its general good solubility of drug-like compounds and high boiling point. 


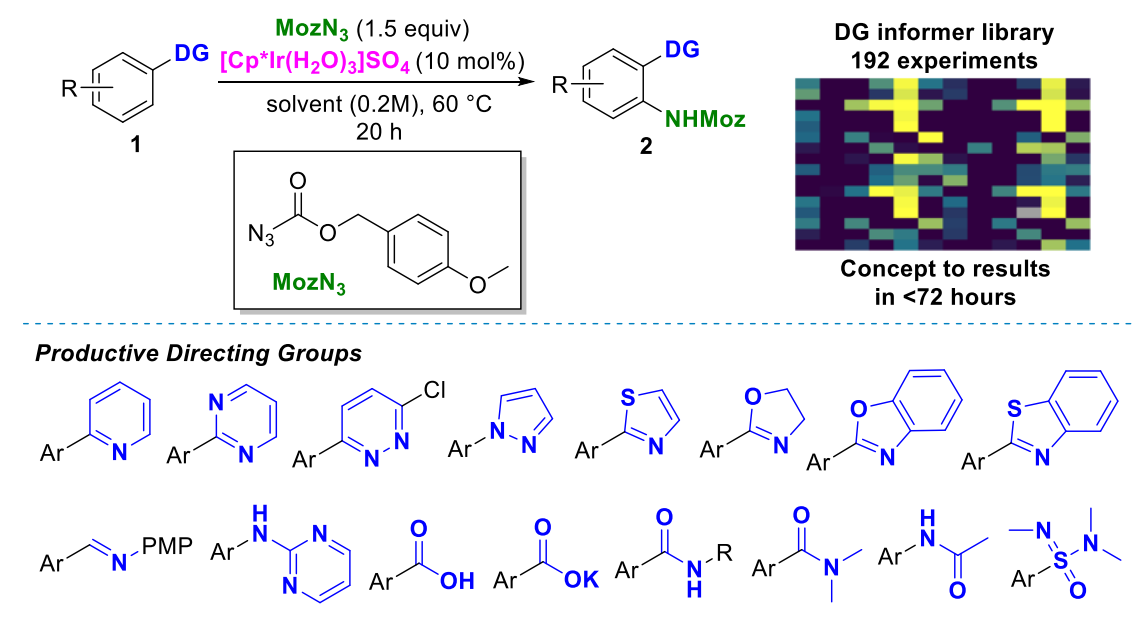

Key Unproductive Directing Groups

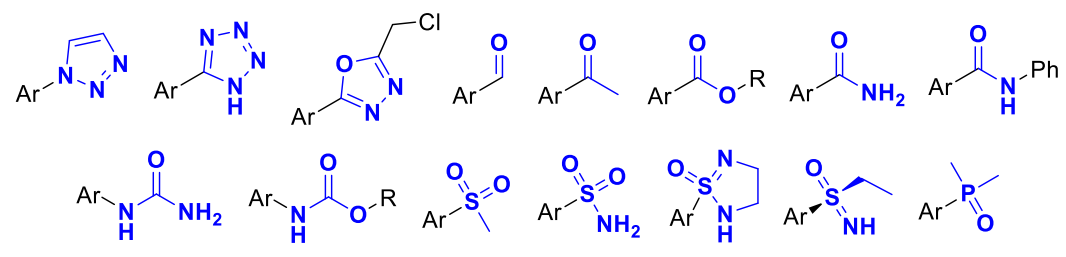

Figure 3. Directing group informer library. 48 substrates tested against four solvents under screening conditions. Productive DGs and key classes of unproductive DGs depicted.

In step 2 (Figure 2), the DG chemical space was probed. Out of the 48 substrates tested under the screening conditions, $16 \mathrm{DG}$ s were shown to be productive for the $\mathrm{C}-\mathrm{N}$ bond formation, with observed conversions ranging from 10 to $>99 \%$ (Figure 3, for more details see SI). Given the variety of DGs tested this was an encouraging result. Although relatively low at the bottom end, we anticipated that the conversions could be improved by further optimization at a later stage. The following observations were made: DCE showed best performance throughout the scope, EtOAC and CPME had similar applicability, albeit in some cases with lower conversions. NMP performed well with heterocycles and carboxylic acids, however, was unproductive with the amide series. While the screening conditions allowed for the functionalization of a variety of substrates under a unified set of conditions, the HTE approach also facilitated rapid substrate-specific reaction optimization. Representative examples are discussed herein (for more studies see SI).

Table 1. Optimization: Catalyst loading and solvent effect.

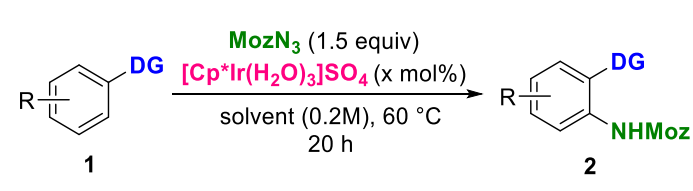

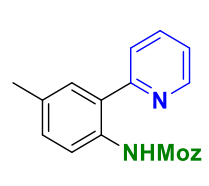

2c

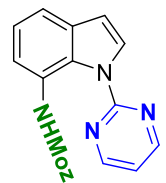

2h

\begin{tabular}{c|cccc|cccc}
\hline Product & \multicolumn{4}{|c|}{ 2c (\%) } & \multicolumn{4}{c}{ 2h (\%) } \\
\hline Ir (mol\%) & NMP & DCE & EtOAC & CPME & NMP & DCE & EtOAc & CPME \\
\cline { 2 - 9 } 10 & 90.9 & 99.1 & 99.5 & 99.5 & 100 & 100 & 100 & 93.6 \\
8 & 92.7 & 99.2 & 100 & 99.4 & 98.0 & 100 & 100 & 83.9 \\
6 & 88.1 & 99.1 & 64.4 & 98.6 & 87.0 & 100 & 96.0 & 66.3 \\
4 & 57.4 & 99.5 & 25.1 & 65.4 & 38.9 & 100 & 81.6 & 35.2 \\
2 & 13.1 & 83.2 & 11.1 & 21.8 & 6.9 & 55.0 & 54.7 & 7.6
\end{tabular}

Ranked by conversion to product. Color coding: red to green, lowest to highest conversion. Analyzed by LCMS (UV trace). 
In the catalyst loading study (Table 1) we observed excellent conversions in all solvents with $10 \mathrm{~mol} \%$ catalyst loading, following the informer library conditions. Performance at lower catalyst loadings was shown to be highly solvent dependent. The best results for both model substrates were obtained with DCE, with excellent conversions even at 4 mol\% catalyst loading. The solvent effect varied between the model substrates, as the second best result for $\mathbf{2 c}$ was obtained in CPME, while for $\mathbf{2} \mathbf{h}$ this was in EtOAc, both at 6 mol\% catalyst loading. The combined effect of catalyst and $\mathrm{MozN}_{3}$ loading on conversion was investigated next (Table 2). A clear trend emerged from this study: while slight excess of $\mathrm{MozN}_{3}$ led to improved conversion, larger excess had a detrimental effect. This was most pronounced at lower catalyst loadings. Catalyst loading at $4 \mathrm{~mol} \%$ and $\mathrm{MozN}_{3}$ ( 1.3 equiv) was chosen for scale-up.

Table 2. Optimization: Catalysts and azide loading effect.

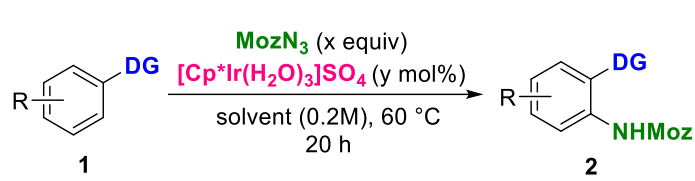

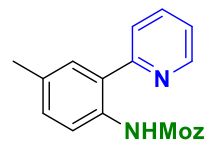

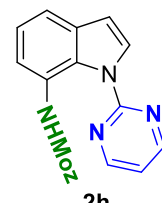

$2 h$

\begin{tabular}{|c|c|c|c|c|c|c|c|c|c|c|c|c|c|}
\hline \multirow{2}{*}{\multicolumn{2}{|c|}{$\begin{array}{l}\text { Product } \\
\text { Solvent }\end{array}$}} & \multicolumn{6}{|c|}{ 2c (\%) } & \multicolumn{6}{|c|}{ 2h (\%) } \\
\hline & & \multicolumn{3}{|c|}{ DCE } & \multicolumn{3}{|c|}{ NMP } & \multicolumn{3}{|c|}{ DCE } & \multicolumn{3}{|c|}{ NMP } \\
\hline $\operatorname{Ir}(r$ & |\%) & 2 & 4 & 6 & 4 & 6 & 8 & 2 & 4 & 6 & 4 & 6 & 8 \\
\hline \multirow{6}{*}{ 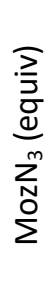 } & 1.0 & 77.9 & 84.7 & 83.5 & 53.1 & 88.0 & 89.0 & 63.6 & 86.7 & 85.7 & 20.1 & 62.3 & 94.9 \\
\hline & 1.1 & 75.1 & 91.6 & 92.3 & 47.7 & 94.0 & 95.1 & 73.9 & 93.3 & 97.1 & 60.1 & 90.2 & 89.8 \\
\hline & 1.3 & 80.1 & 100 & 100 & 46.6 & 88.4 & 94.1 & 71.0 & 100 & 100 & 60.7 & 90.0 & 90.7 \\
\hline & 1.5 & 71.0 & 100 & 100 & 53.8 & 85.8 & 90.9 & 66.3 & 100 & 100 & 42.1 & 87.7 & 90.5 \\
\hline & 2.0 & 57.2 & 98.0 & 100 & 47.4 & 70.5 & 88.0 & 58.6 & 100 & 100 & 20.7 & 58.2 & 87.3 \\
\hline & 3.0 & 53.4 & 86.6 & 100 & 44.9 & 68.6 & 86.2 & 42.6 & 91.4 & 100 & 25.0 & 60.3 & 82.4 \\
\hline
\end{tabular}

Ranked by conversion to product. Color coding: red to green, lowest to highest conversion. Analyzed by LCMS (UV trace).

As formation of mono- and difunctionalization mixtures was observed for a number of compounds in the directing group informer library, tunable selectivity was investigated with 1-phenyl-1H-pyrazole (1e) as model system (Table 3). Good monoselectivity was achieved at low catalyst and $\mathrm{MozN}_{3}$ loading. Conversely, enrichment of the difunctionalization product was achieved with increased catalyst and azide loadings.

Table 3. Optimizing: Mono/di selectivity.

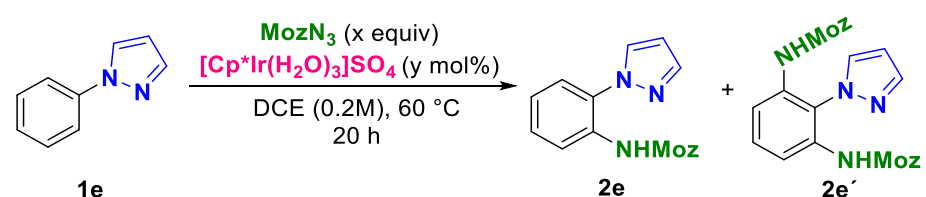

\begin{tabular}{|c|c|c|c|c|c|c|c|}
\hline \multirow{2}{*}{\multicolumn{2}{|c|}{$\begin{array}{l}\text { Ir (mol\%) } \\
\text { Product }\end{array}$}} & & \multicolumn{2}{|c|}{4} & \\
\hline & & $2 \mathrm{e}(\%)$ & $2 e^{\prime}(\%)$ & $2 e(\%)$ & $2 e^{\prime}(\%)$ & $2 e(\%)$ & $2 e^{\prime}(\%)$ \\
\hline \multirow{5}{*}{ 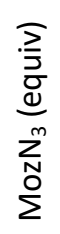 } & 1.0 & 86.4 & 9.5 & 86.9 & 9.4 & 86.9 & 10.2 \\
\hline & 1.1 & 85.9 & 10.4 & 86.8 & 7.7 & 85.6 & 13.0 \\
\hline & 1.3 & 78.0 & 21.2 & 77.6 & 22.1 & 73.7 & 26.3 \\
\hline & 1.5 & 81.1 & 17.5 & 53.9 & 45.8 & 49.6 & 50.1 \\
\hline & 2.0 & 84.3 & 10.6 & 53.4 & 46.0 & 22.7 & 76.4 \\
\hline
\end{tabular}

Ranked by conversion to product. Color coding: red to green, lowest to highest conversion. Analyzed by LCMS (UV trace). 
To confirm the performance of the catalytic system on a larger scale, a series of building blocks were functionalized and isolated (Scheme 1). The building block selection was based on positive hits from the directing group informer library (vide supra, Figure 3). The effect of various substitution patterns on the functionalized system was investigated with the 2-phenylpyridine series. While the orthomethyl substituent was well tolerated in $\mathbf{1 a}$, with increased substituent size in $\mathbf{1 b}$ a significant decrease in yield was observed (2b, $\mathbf{3 7 \%}$ ). The meta substituent in $\mathbf{1 c}$ was tolerated and yielded the anticipated product (2c) with complete regioselectivity. Importantly, the reaction could also be performed using CPME as solvent with maintained yield, albeit with increased catalyst loading (6 mol\%). Functionalization of 2-phenylpyridine with high monoselectivity was achieved with decreased $\mathrm{MozN}_{3}$ loading (for optimization see $\mathrm{SI}$ ), yielding compound $\mathbf{2 d}$ in $86 \%$ yield. The monoselective functionalization observed with pyrazole $1 \mathbf{e}$ in the optimization study (Table 3 ) translated well to the

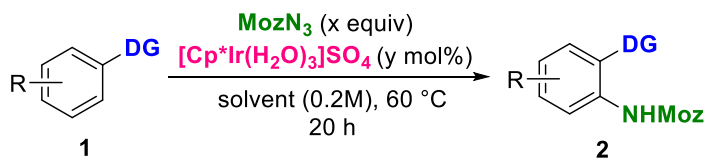

Scale up (0.5 mmol)

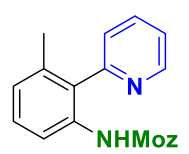

2a, $76 \%$

Ir (4 mol\%)

$\mathrm{MozN}_{3}$ (1.3 equiv)

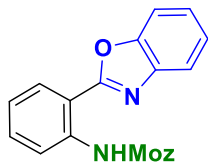

2f, $40 \%^{a}$

Ir (6 mol\%) $\operatorname{MozN}_{3}$ (1.0 equiv)

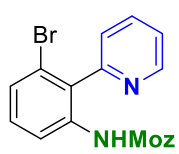

2b, $37 \%$

Ir (4 mol\%)

$\operatorname{MozN}_{3}$ (1.3 equiv)

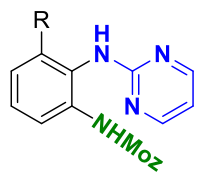

2g, R: $\mathrm{H}, 46 \%^{b}$

2g', R: NHMoz, 7\%

Ir (10 mol\%)

$\mathrm{MozN}_{3}$ (1.5 equiv)<smiles>CC(=O)Nc1ccccc1NC(C)=O</smiles>

2k, $25 \%^{b}$

Ir (10 mol\%)

$\mathrm{MozN}_{3}$ (1.0 equiv)

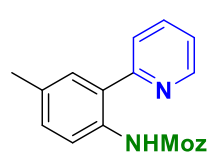

2c, $88 \%, 88 \%{ }^{\circ}$

Ir (4 mol\%)

$\mathrm{MozN}_{3}$ (1.3 equiv)

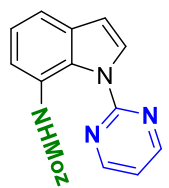

2h, $40 \%^{a}, 41 \%^{a, d}$

Ir (4 mol\%)

$\mathrm{MozN}_{3}$ (1.3 equiv)<smiles>CN(C)C(=O)c1ccccc1N=O</smiles>

2I, $53 \%^{a}$

Ir (10 $\mathrm{mol} \%)$

$\operatorname{MozN}_{3}$ (1.3 equiv)

$\mathrm{C}_{5} \mathrm{H}_{9} \mathrm{COOH}$ (1.0 equiv)

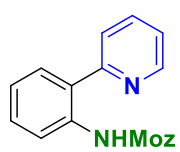

2d, $86 \%$

Ir (4 mol\%)

$\operatorname{MozN}_{3}$ (1.05 equiv)

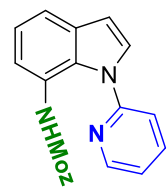

2i, $83 \%^{a}$

Ir (4 mol\%)

$\operatorname{MozN}_{3}$ (1.3 equiv)<smiles>CON(C)C(=O)c1ccccc1N=O</smiles>

$2 \mathrm{~m}, 28 \%^{a}$

Ir (10 mol\%)

$\mathrm{MozN}_{3}$ (1.3 equiv)

$\mathrm{C}_{5} \mathrm{H}_{9} \mathrm{COOH}$ (1.0 equiv)

\section{Screening scale $(0.02 \mathrm{mmol})^{e}$}

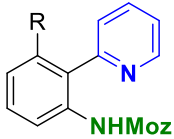

2d, R: H, $37 \%$

2d', R: NHMoz, 8\%

Solvent: EtOAc

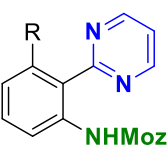

2q, R: H, 7\%

2q', R: NHMoz, 32\%

Solvent: NMP

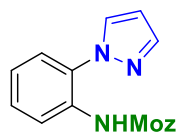

2e, R: $\mathrm{H}, 46 \%$ 2e', R: NHMoz, 10\% Solvent: CPME<smiles>[R]c1cccc(Nc2c([R])cccc2-c2ccc(Cl)nn2)c1</smiles>

2r, R: H, $8 \%$

2r', R: NHMoz, 29\% Solvent: NMP<smiles>CNc1ccccc1C1=NCCO1</smiles>

2n, $47 \%$ Solvent: DCE<smiles></smiles>

2s, $16 \%$

Solvent: DCE<smiles>CNc1ccccc1-c1nccs1</smiles>

2o, $25 \%$

Solvent: DCE

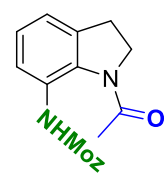

2t, $16 \%$

Solvent: CPME

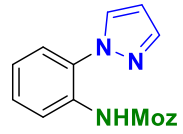

2e, $76 \%$

Ir (2 mol\%)

$\mathrm{MozN}_{3}$ (1.0 equiv)

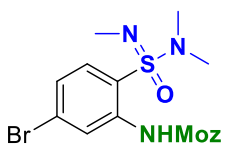

2j, $34 \%^{b}$

Ir (10 mol\%) $\mathrm{MozN}_{3}$ (1.5 equiv)

Scheme 1. Scope: Building blocks. Directing groups highlighted in blue. Isolated yields shown. ${ }^{a}$ Reaction scale $0.2 \mathrm{mmol} .{ }^{b}$ Reaction Scale $0.1 \mathrm{mmol} .{ }^{c}$ CPME as solvent. ${ }^{d}$ EtOAc as solvent. ${ }^{e}$ isolated from DG informer library reaction plate. 
$0.5 \mathrm{mmol}$ scale. Benzoxazole $\mathbf{2 f}$ was successfully obtained under modified conditions based on single substrate optimization results (see $\mathrm{SI}$ ). The utility of the presented catalytic system beyond the formation of 5-membered iridacycles was demonstrated with the $\mathbf{2 g}-\mathbf{2} \mathbf{i}$ series, yielding the desired products via 6-membered iridacycle formation. The reaction of $\mathrm{N}$-phenylpyrimidin-2-amine yielded a mixture of monoaminated $\mathbf{2} \mathbf{g}$ and diaminated $\mathbf{2} \mathbf{g}^{\prime}$, favoring the monofunctionalization product. In the indole series, both $\mathbf{2 h}$ and $\mathbf{2 i}$ were obtained with complete selectivity for the 7-position over the 2position, favoring 6 -membered iridacycle formation. Importantly with $\mathbf{2 h}$, the use of more environmentally benign EtOAc as solvent had no negative effect on yield. To our delight oxygencentered directing groups were also successfully utilized as demonstrated with the $\mathbf{2} \mathbf{j}-\mathbf{2} \mathbf{m}$ series. Sulfonimidamides are an emerging class of compounds within medicinal chemistry, ${ }^{40}$ and to the best of our knowledge, the synthesis of $\mathbf{2} \mathbf{j}$ presents the first application of this moiety within directed $\mathrm{C}-\mathrm{H}$ activation. Functionalization of acetanilide $\mathbf{2 k}$ extends the accessibility of 6 -membered iridacyles to oxygen-centered directing groups. Compound $\mathbf{2 l}$ was obtained with improved yield by adding cyclopentane carboxylic acid as additive. Improvement of conversion with amide directing groups in combination with carboxylic acid additives was observed during the LSF scope investigation with Bezafibrate 3j (Scheme 2). This observation further extended the scope of accessible directing groups with Weinreb amides, as shown with $\mathbf{2} \mathbf{m}$. This substrate class was unproductive under screening conditions of the directing group informer library (Figure 2). While reaction scale-up was vital for further applications, we were also interested in extending the utility of screening libraries by product isolation from small-scale reactions. At $0.02 \mathrm{mmol}$ reaction scale, the products of a series of 10 building blocks were isolated in quantities sufficient for characterization by NMR spectroscopy. The potential utility of small-scale reaction substance isolation extends beyond compound characterization, as a single milligram of compound is often sufficient for in depth biological studies.$^{41} \mathrm{~A}$ common set-back of this approach are reduced product yields due to sample handling and purification-associated loses, as demonstrated with the decreased yields of $\mathbf{2} \mathbf{d}$ and of $\mathbf{2 e}$ (Scheme 1, top vs. bottom). The applicability of heterocyclic DGs was further demonstrated with dihydrooxazole $\mathbf{1 n}$, thiazole $\mathbf{1 0}$, benzothiazole $\mathbf{1 p}$, pyrimidine $\mathbf{1 q}$ and pyridazine $\mathbf{1 r}$. The PMP-capped imine $\mathbf{2 s}$ was obtained with low yield as a result of hydrolysis during purification. Products from oxygen-centered directing groups in $\mathrm{N}$-acetyl indoline $\mathbf{2 t}$ and $\mathrm{N}$-methyl benzamide $\mathbf{2} \mathbf{u}$ were also isolated, the latter with lower yield due to problematic separation from unreacted starting material. 
Table 4. Functional group compatibility study.

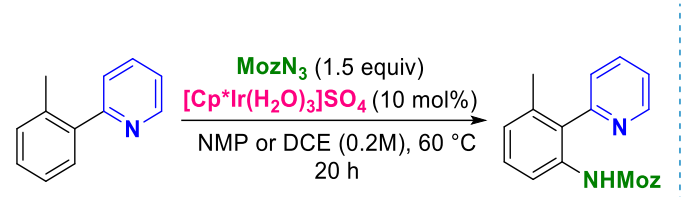

$1 \mathrm{a}$
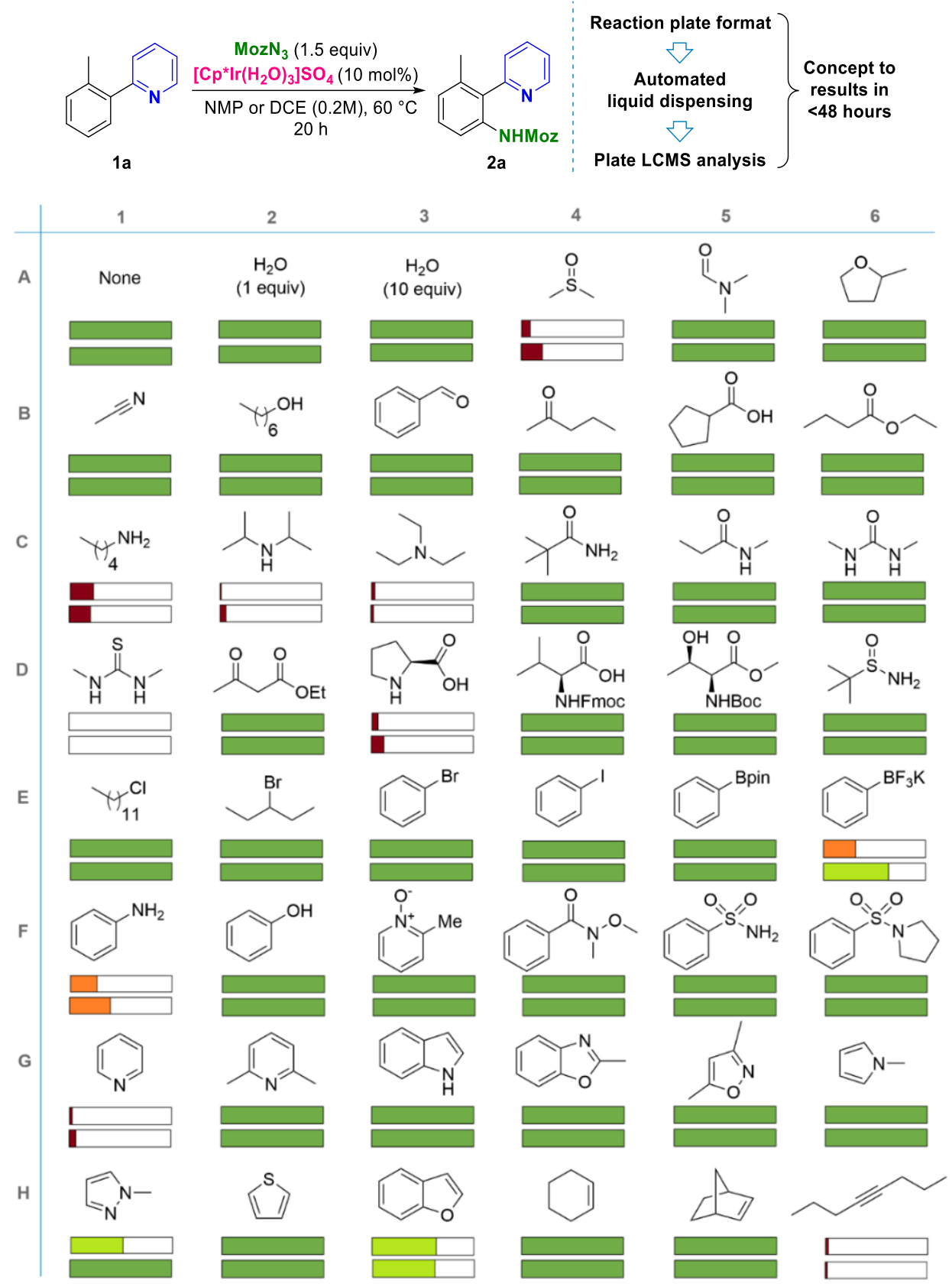

Reaction solvents: NMP (top bars), DCE (bottom bars). One equivalent of additive used per reaction. Color coding based on conversion: Green $>50 \%$, orange $50-25 \%$, red $<25 \%$. Analyzed by LCMS (UV trace).

In step 3 (Figure 2), the effect of a series of 46 additives on reaction performance was evaluated (Table 4). The additives were chosen as a means of representing functional groups commonly present in druglike molecules. ${ }^{30,33,34}$ In terms of solvent effect, only minimal differences in performance between NMP (top bars) and DCE (bottom bars) were observed. To our delight, out of 47 modified conditions, 35 had no effect on the reaction outcome. Notably, excess water was well tolerated for the catalytic conditions used here, a feature important for use of reagents without prior drying. In terms of reagent and functional group tolerance the following insights were gained: 1) the presence of DMSO has a negative effect on the reaction outcome, while other commonly used polar and/or protic solvents are well tolerated. This observation is in accordance to similar studies. ${ }^{33}, 342$ ) The majority of polar 
functional groups commonly present in drug-like molecules were well tolerated. This includes the ether, alcohol, phenol, aldehyde, ketone, carboxylic acid, ester, primary and secondary amides, urea, Weinreb amide and sulfonamides. The same was observed with functional groups commonly present in cross-coupling reagents, such as aliphatic and aromatic halides and aryl-Bpin group. 3) The utility of the method is limited by the presence of amines; with primary, secondary and tertiary amines. The presence of aniline leads to significant decrease in conversion. 4) While heterocycles are in general well tolerated, the presence of pyridine is detrimental. The activity is restored by sterically hindering the pyridine nitrogen (pyridine vs. 2,6-lutidine). 5) Alkenes are tolerated, but the presence of alkynes leads to complete inhibition of the reaction. While this approach provides valuable information on the limitations of this methodology, we recognize that such a simplified approach has its limitations, as the integrity of the additives post-reaction was not determined. Changes in electronic properties of the additives by substituents variation can also effect compatibility.

In step 4 (Figure 2), we directed our attention to late-stage amination of a set of complex molecules, consisting of small molecule drugs and natural products. The value of the LSF informer library builds on the investigations presented so far, as it introduces further complexity resulting from the interplay of multiple functional groups in a single substrate. A 48 membered LSF informer library was used, this time tested against two solvents, NMP and DCE. Out of these, 11 were considered successful (conversion $>10 \%$ ), with structures confirmed by NMR spectroscopy. Further 4 compounds were not considered successful due to low conversion and/or product decomposition during purification. The value of performing step 2 (DG informer library) and step 3 (functional group tolerance study) of the envisioned workflow (Figure 2) was shown already at this stage. Out of the 33 remaining compounds, 14 contained unproductive directing groups and 9 contained amines in their structure. The cause behind the failure of the remaining 10 substrates is proposed to be a combination of steric effects, combination effects of functional groups and presence of unproductive directing groups not included in the directing group informer library.

Although the reaction conditions for the substrates were mostly based on findings from building blocks reaction optimizations, single substrate optimization also proved of high utility for LSF examples (see $\mathrm{SI})$. In case of Atazanavir, a single reaction conditions screening allowed us to rapidly identify optimal conditions for accessing the monofunctionalized product 3a and difunctionalized product 3a' with high degree of selectivity in good to very good yields. Further worth noting is the compatibility of the reaction conditions with a number of polar and protic groups, including arrangements of functional groups suitable for bidentate coordination in the peptide backbone. The pyridine moiety also served as a suitable directing group in Priletivir. Compound $\mathbf{3 b}$ was successfully obtained, with the primary sulfonamide, thiazole and tertiary amide groups tolerated. The heavily substituted pyrazole of Apixaban served as a productive directing group, further demonstrating the utility of this moiety for directed $\mathrm{C}-\mathrm{H}$ amination. Important noting is the use of NMP as the reaction solvent, as the substrate was insoluble in DCE. While the reaction offered relatively low isolated yield of $\mathbf{3 c}$, the majority of 

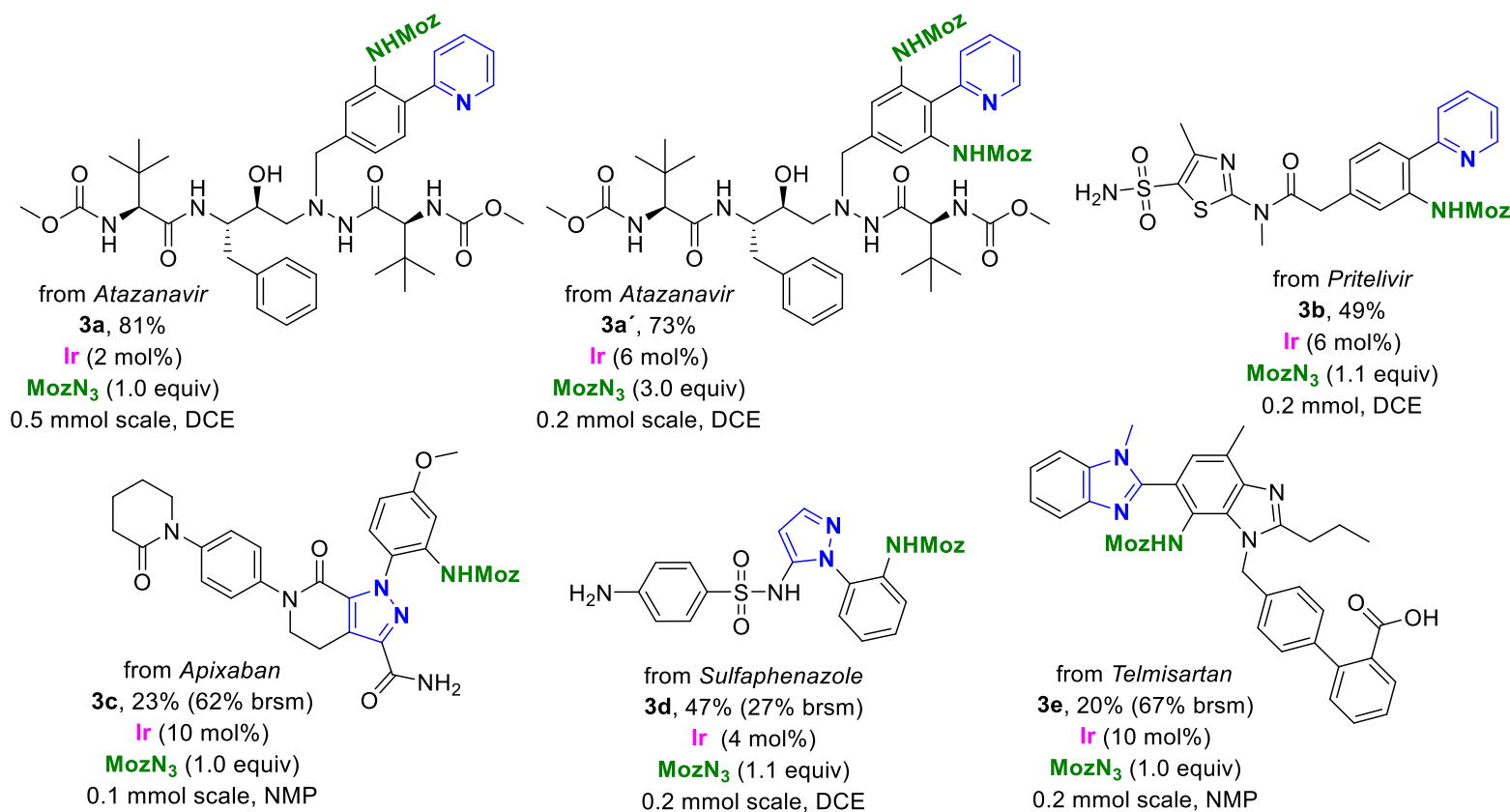

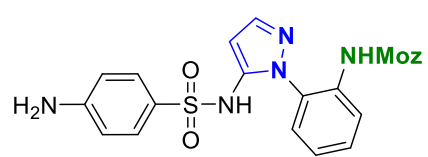

from Sulfaphenazole 3d, $47 \%(27 \%$ brsm $)$ Ir $(4 \mathrm{~mol} \%)$

$\mathrm{MozN}_{3}$ (1.1 equiv)

$0.2 \mathrm{mmol}$ scale, DCE

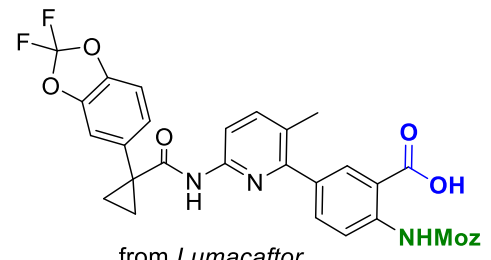

from Lumacaftor

$\mathbf{3 g}, 16 \%(74 \%$ brsm $)$

Ir (10 mol\%)

$\operatorname{MozN}_{3}$ (1.0 equiv)

$0.1 \mathrm{mmol}$ scale, NMP

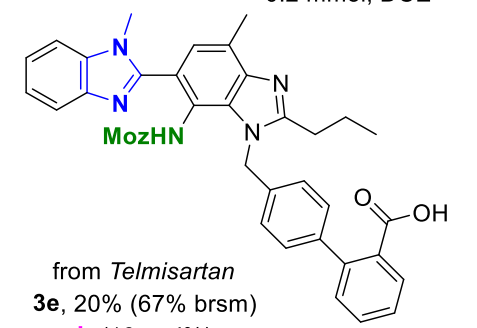

Ir (10 mol\%)

$\mathrm{MozN}_{3}$ (1.0 equiv)

$0.2 \mathrm{mmol}$ scale, NMP

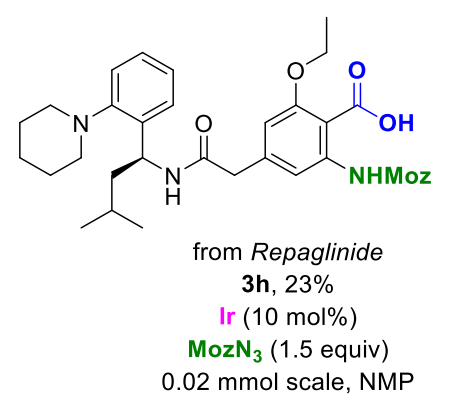

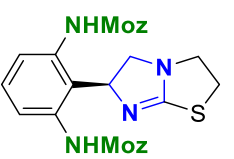

from Levamisole $3 \mathbf{k}, 32 \%$ $\operatorname{Ir}(10 \mathrm{~mol} \%)$ $\mathrm{MozN}_{3}$ ( 1.5 equiv) $0.1 \mathrm{mmol}, \mathrm{DCE}$

Scheme 2. Scope: LSF. Directing groups highlighted in blue. Isolated yields shown.

unreacted starting material was successfully isolated. In the case of Sulfaphenazole, the pyrazole moiety bearing a sulfonamide group in the 5 position served as a suitable directing group, yielding analogue $3 \mathbf{d}$. Worth noting is the improved tolerance of the aniline compared to the functional group tolerance study (Table 4), presumably due to different electronic properties of the nitrogen affected by the para sulfonamide group. A case of unexpected selectivity was observed with Telmisartan, where product $3 \mathbf{e}$, resulting from the coordination of the $\mathrm{N}$-methylbenzimidazole, was obtained as a single regioisomer, with no product formation observed from the carboxylate coordination. This is also the only example where we observed the formation of product with a 1,2,3 substitution pattern. We rationalize the observed selectivity as a result of the steric arrangement of the substrate, with the ortho substituent of the benzoic acid moiety decreasing its reactivity, and the fused ring system on the 3 position decreasing steric hindrance and allowing functionalization in a 1,2,3 layout. The imine moiety of Diazepam facilitated the amination of the phenyl core. Both monofunctionalized $\mathbf{3 f}$ and difunctionalized $\mathbf{3} \mathbf{f}^{\prime}$ were successfully isolated. It is important to note that the products were isolated 
directly from the LSF informer library at $0.02 \mathrm{mmol}$ scale, in quantities sufficient for complete characterization. Lumacaftor was successfully functionalized in NMP with complete selectivity for the carboxylate-directed amination. While with relatively low conversion, the 2-acylanilido pyridine structural motif was tolerated. A significant amount of unreacted starting material was also recovered. The product of carboxylate-directed $\mathrm{C}-\mathrm{H}$ amination of Repaglinide $3 \mathrm{~h}$ was successfully isolated on the $0.02 \mathrm{mmol}$ screening scale. The tolerance of the tertiary amine moiety is rationalized by its anilinic nature. A powerful example of the utility of the herein described amination protocol is demonstrated with the functionalization of Paclitaxel. This complex natural product contains a number polar and protic functional groups, sensitive ester groups, a strained oxetane ring and an unsaturation, all of which pose a potential challenge for LSF methods. While $3 \mathbf{i}$ could be obtained with $20 \%$ isolated yield under standard conditions, the use of one equivalent of acid additive allowed for increasing the isolated yield to $72 \%$. This result, to the best of our knowledge, presents the highest yielding example of Paclitaxel $\mathrm{C}-\mathrm{H}$ functionalization reported to date. The observation of the positive effect of carboxylic acid additives on conversions with substrate bearing amide directing groups was first observed with the example of Bezafibrate. The conversion to $\mathbf{3} \mathbf{j}$ was much higher than conversions of corresponding amides in the DG informer library. This unexpected observation further strengthens the case for LSF informer libraries, as the combinations of structural motifs directly aided methodology development. Finally, Levamisole, bearing an unusual $\mathrm{sp}^{2}$-sp $\mathrm{p}^{3}$ linkage between the benzene core and the directing group, was selectively difunctionalized to yield product 3k. A point worth noting is that even though the isolated yields for a number of presented examples were relatively low, in many cases these may still be comparable or exceeding expected overall yields from de novo synthesis of these analogues. The amount of material obtained from these reactions would suffice for the needs of biological studies, allowing for rapid access to SAR data in a fraction of time compared to de novo synthesis.

\section{Deprotection studies}
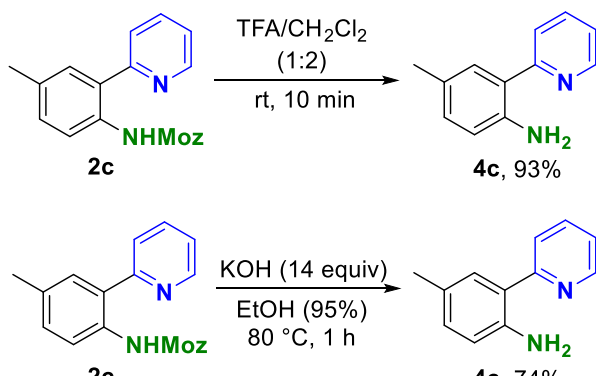

2c

4c, $74 \%$

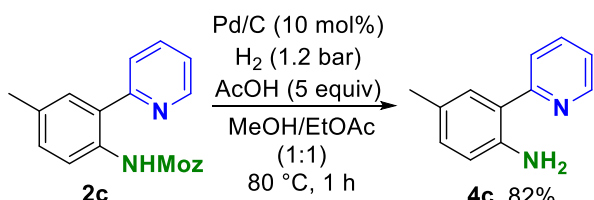
$80^{\circ} \mathrm{C}, 1 \mathrm{~h}$

4c, $82 \%$

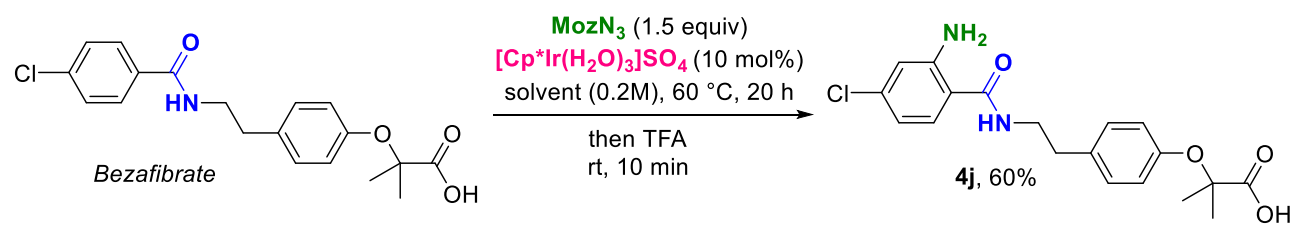

Scheme 3. Deprotection studies. Isolated yields shown.

The Moz group was successfully deprotected with three distinct deprotection protocols. This is of particular importance in terms of LSF applications, allowing for deprotection conditions tolerant of a wide array of functional groups. Deprotection under acidic conditions in presence of TFA yielded the corresponding aniline in excellent yields. The desired compound was also obtained under basic 
condition, using excess $\mathrm{KOH}$ in refluxing $\mathrm{EtOH}$. In the third protocol, hydrogenolysis using standard $\mathrm{Pd} / \mathrm{C}$ hydrogenation yielded the $4 \mathrm{c}$ product in very good yield. Finally, LSF application of a one-pot amination/deprotection protocol was demonstrated with Bezafibrate, yielding the free aniline product $4 \mathbf{j}$ in $60 \%$ isolated yield.

\section{Miniaturization studies}

In the final experimental study we further investigated the possibilities in reaction miniaturization enabled by the use of NMP as non-volatile solvent. We found that with as little as one microliter of

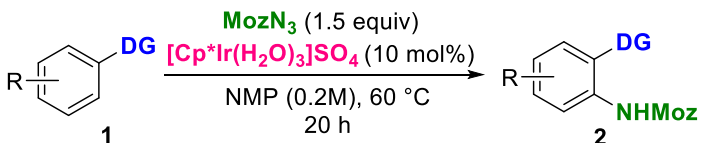
2

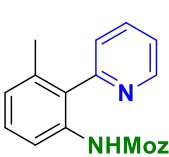

2a, Success rate: $12 / 16$ Conversion range: $48.5-100 \%$ Conversion avg.: $91.9 \%$

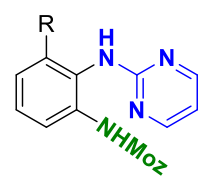

$\mathbf{2 g}, \mathbf{2} \mathbf{g}^{\prime}$, Success rate: $14 / 16$ Conversion range (2g): 26.4 - 44.9 Conversion avg. $(\mathbf{2 g}): 36.7 \%$ Conversion range $\left(\mathbf{2 g}^{\prime}\right): 9.0-21.7 \%$ Conversion avg. $\left(\mathbf{2} \mathbf{g}^{\prime}\right): 15.9$

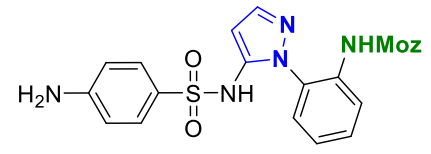

from Sulfaphenazole 3d Success rate: $15 / 16$ Conversion range: $34.2-59.1 \%$ Conversion avg.: $91.9 \%$<smiles>[CH]Cc1cc(CN(C[C@@H](O)[C@@H](Cc2ccccc2)NC(=O)[C@@H](NC(=O)OC)C(C)(C)C)NC(=O)C(NC(=O)OC)C(C)(C)C)ccc1-c1ccccn1</smiles>

from Atazanavir

Conversion avg.: $100 \%$

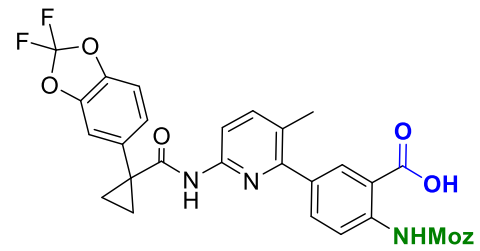

rom Lumacaftor

$\mathbf{3 g}$, Success rate: $11 / 13$ Conversion range: 26.9 - $37.9 \%$

Conversion avg.: $33.0 \%$

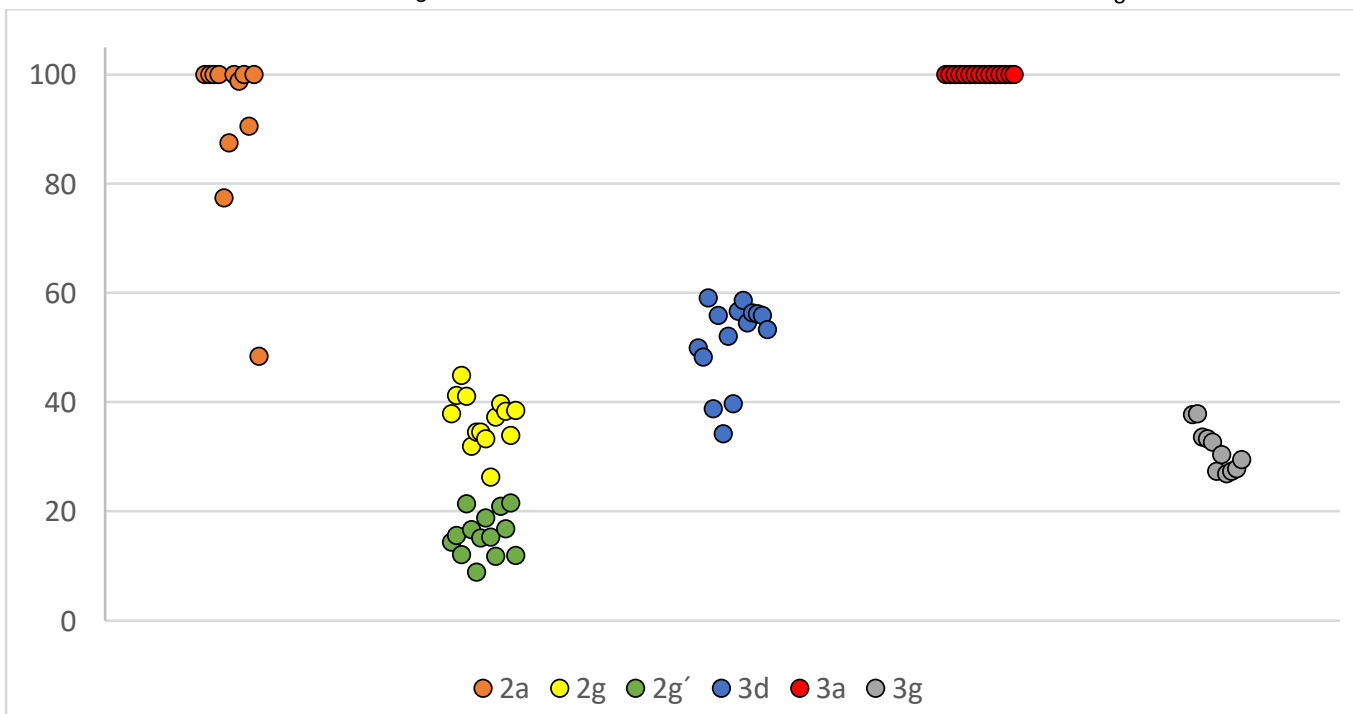

Figure 4. Miniaturization study. Reaction scale $200 \mathrm{nmol}$, reaction volume $1.0 \mu \mathrm{L}$. Analyzed by LCMS (UV trace). With unsuccessful reaction no substrate or product was detected.

total reaction volume conversion to the anticipated products with reasonable reproducibility was obtained throughout the selected substrates (Figure 4). To the best of our knowledge, this presents the smallest scale $\mathrm{C}-\mathrm{H}$ activation reported to date, and presents an exciting opportunity in terms of improved sustainability of reaction screening by decreased material consumption. We were even able 
to further scale down the reaction by using acoustic dispensing for setting up the reaction plates. ${ }^{42}$ With this technique, we were able to detect product formations in reaction with a total volume as little as $5 \mathrm{~nL}$ ( $1 \mathrm{nmol}$ scale). Taking Sulfaphenazole as an example, this means that from one gram of material 3181 reactions can be performed. Chemistry at this small scale brings its own set of challenges, and at this point we were not able to quantify conversions with reasonable accuracy and throughput. We are currently working on an AMI-MS analytical method to tackle this issue. ${ }^{43}$

\section{Application guidelines}

In the final part of this work, step 5 (Figure 2), we present guidelines for reaction outcome prediction. (Figure 5).

1) Directing group selection. In total 21 productive directing groups were presented in the DG and LSF informer libraries, as well as 20 non-productive directing groups from the DG informer library (Figure 18 , for complete substrate structures see SI).

2) Determination of tolerated functional groups. This is aided by the functional group tolerance study and LSF scope. The major limitations in this respect are presence of amines, alkynes, thioureas, and residual DMSO. The DMSO sensitivity is important to consider in medicinal chemistry applications, as intermediates are often stored as DMSO solutions and residual solvent can be present after evaporation.

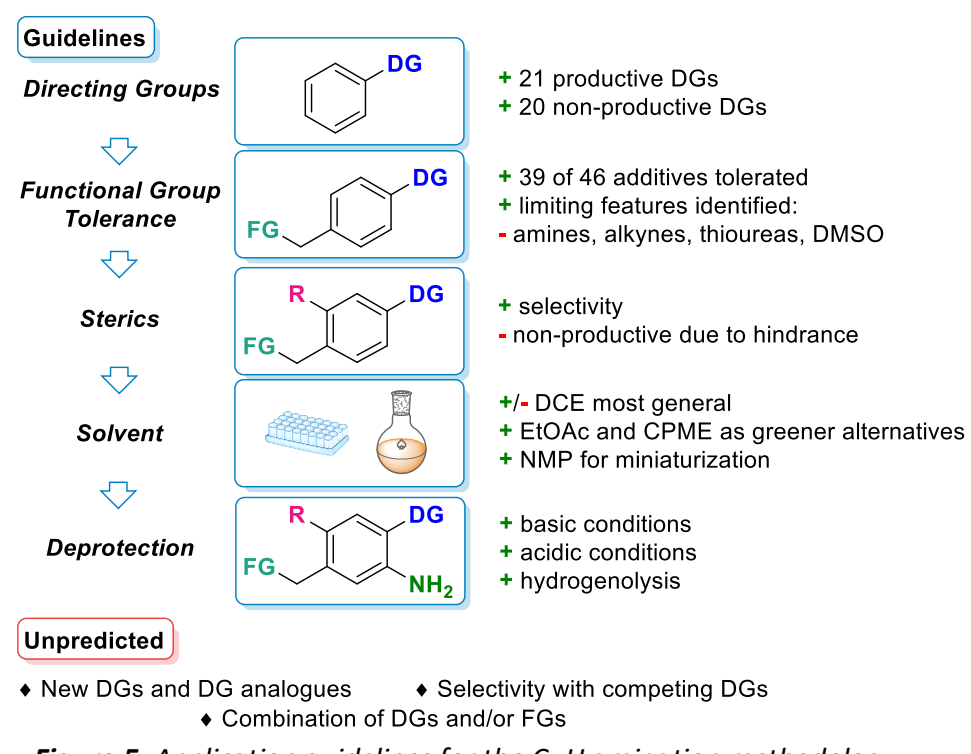

3) Steric effects on the substrate should be examined in order to determine selectivity and/or productivity. Based on the observed results, the reaction proceeds on the less sterically hindered ortho position when two suitable reaction sites are available. Meta substitution with sterically demanding substituents blocks 1,2,3-substitution. Substituents on the directing group and in the ortho position of the system to be functionalized can negatively affect the reaction outcome by twisting the directing group out of plane. ${ }^{44}$

4) Reaction solvent is chosen based on desired application. While DCE showed best overall performance, substitution by EtOAc and CPME is possible if the use of a greener solvent is desired. The use of NMP is ideal for applications in miniaturization, as well as for applications with polar drug-like molecules displaying low solubility in DCE.

5) The final step is the choice of deprotection conditions. The range of presented protocols should satisfy the needs of potential applications in LSF.

Although we studied the reaction extensively, the predictive model has its limitations. Given the breadth of the chemical space of Lewis-basic DGs, it is likely that some DGs and their analogues remain 
uninvestigated. Selectivity between DGs, while observed in most cases, was not extensively investigated. Finally, while the functional group tolerance study provides basic guidelines, the combination effect of functional groups, or even combination effects with DGs are not possible to predict.

\section{Conclusion}

A directed iridium-catalyzed $\mathrm{C}-\mathrm{H}$ amination methodology applicable to substrates with a wide range of directing groups and with outstanding functional group tolerance was developed. HTE applications facilitated not only rapid optimization of reaction conditions, but also allowed for reaction miniaturization to nanomolar scale and use of automation throughout the campaign. An important aspect of this study was exploring both the opportunities and limitations of the reaction, disclosing both successful and unsuccessful reactions. The directing group and LSF informer libraries, in combination with the functional group tolerance studies allowed for the generation of guidelines for predicting reaction applicability to complex substrates. In terms of demonstrated substrate scope, a broad range of building blocks with diverse directing groups were synthesized, and late-stage functionalization of a number of structurally complex drugs and natural products was demonstrated. The utility of the presented method for applications on complex substrates is further increased by access to a range of Moz deprotection protocols. We are confident that the presented method and associated techniques will find applications in other laboratories. Finally, it is our sincere hope that this work will inspire others to disclose the limitation of their methodologies, allowing users to save time and effort on unproductive reactions, but also eliminating material consumption for such reactions, and ultimately reducing the environmental impact of synthetic chemistry.

\section{Acknowledgements}

E.W. is supported by the Industrial PhD Student scheme from the Swedish Foundation for Strategic Research (SSF). The authors are grateful for support from the Swedish Research Council through Vetenskapsrådet, from the Swedish Foundation for Strategic Environmental Research (Mistra SafeChem, project number 2018/11) and from the Göran Gustafsson Foundation.

\section{References}

1. D. C. Blakemore, L. Castro, I. Churcher, D. C. Rees, A. W. Thomas, D. M. Wilson and A. Wood, Nat. Chem., 2018, 10, 383-394.

2. G. Chessari, R. Grainger, R. S. Holvey, R. F. Ludlow, P. N. Mortenson and D. C. Rees, Chem. Sci., 2021, 12, 11976-11985

3. T. Cernak, K. D. Dykstra, S. Tyagarajan, P. Vachal and S. W. Krska, Chem. Soc. Rev., 2016, 45, 546-576.

4. O. O. Grygorenko, D. M. Volochnyuk, S. V. Ryabukhin and D. B. Judd, Chem. Eur. J., 2020, 26, 1196-1237.

5. S. W. Krska, D. A. DiRocco, S. D. Dreher and M. Shevlin, Acc. Chem. Res., 2017, 50, 2976-2985.

6. B. Mahjour, Y. Shen and T. Cernak, Acc. Chem. Res., 2021, 54, 2337-2346.

7. S. D. Dreher and S. W. Krska, Acc. Chem. Res., 2021, 54, 1586-1596.

8. J. Börgel and T. Ritter, Chem, 2020, 6, 1877-1887.

9. L. Guillemard, N. Kaplaneris, L. Ackermann and M. J. Johansson, Nat. Rev. Chem., 2021, 5, 522-545.

10. T. Rogge, N. Kaplaneris, N. Chatani, J. Kim, S. Chang, B. Punji, L. L. Schafer, D. G. Musaev, J. Wencel-Delord, C. A. Roberts, R. Sarpong, Z. E. Wilson, M. A. Brimble, M. J. Johansson and L. Ackermann, Nat. Rev. Methods Primers, 2021, 1, 43.

11. Y. Park, Y. Kim and S. Chang, Chem. Rev., 2017, 117, 9247-9301.

12. J. Park and S. Chang, Chem. Asian J., 2018, 13, 1089-1102.

13. T. M. Figg, S. Park, J. Park, S. Chang and D. G. Musaev, Organometallics, 2014, 33, 4076-4085. 
14. K. Shin, H. Kim and S. Chang, Acc. Chem. Res., 2015, 48, 1040-1052.

15. M. R. Sk, S. S. Bera, S. Basuli, A. Metya and M. S. Maji, Asian J. Org. Chem., 2020, 9, $1701-$ 1717.

16. S. Sunny and R. Karvembu, Adv. Synth. Catal., 2021, 363, 4309-4331.

17. H.-W. Wang, Y. Lu, B. Zhang, J. He, H.-J. Xu, Y.-S. Kang, W.-Y. Sun and J.-Q. Yu, Angew. Chem. Int. Ed., 2017, 56, 7449-7453.

18. S. Rej and N. Chatani, Angew. Chem. Int. Ed., 2019, 58, 8304-8329.

19. J. Y. Kim, S. H. Park, J. Ryu, S. H. Cho, S. H. Kim and S. Chang, J. Am. Chem. Soc., 2012, 134, 9110-9113.

20. Y. Hwang, Y. Park and S. Chang, Chem. Eur. J., 2017, 23, 11147-11152.

21. D. Lee, Y. Kim and S. Chang, J. Org. Chem., 2013, 78, 11102-11109.

22. D. Lee and S. Chang, Chem. Eur. J., 2015, 21, 5364-5368.

23. H. Kim and S. Chang, ACS Catal., 2015, 5, 6665-6669.

24. H. Kim, G. Park, J. Park and S. Chang, ACS Catal., 2016, 6, 5922-5929.

25. J. Kim, J. Kim and S. Chang, Chem. Eur. J., 2013, 19, 7328-7333.

26. V. S. Thirunavukkarasu, K. Raghuvanshi and L. Ackermann, Org. Lett., 2013, 15, 3286-3289.

27. M. Bhanuchandra, M. Ramu Yadav, R. K. Rit, M. Rao Kuram and A. K. Sahoo, Chem. Commun., 2013, 49, 5225-5227.

28. Q.-Z. Zheng, Y.-F. Liang, C. Qin and N. Jiao, Chem. Commun., 2013, 49, 5654-5656.

29. K. D. Collins and F. Glorius, Acc. Chem. Res., 2015, 48, 619-627.

30. K. D. Collins and F. Glorius, Nat. Chem., 2013, 5, 597-601.

31. T. Gensch and F. Glorius, Science, 2016, 352, 294.

32. P. S. Kutchukian, J. F. Dropinski, K. D. Dykstra, B. Li, D. A. DiRocco, E. C. Streckfuss, L.-C. Campeau, T. Cernak, P. Vachal, I. W. Davies, S. W. Krska and S. D. Dreher, Chem. Sci., 2016, 7, 2604-2613.

33. S. D. Friis, M. J. Johansson and L. Ackermann, Nat. Chem., 2020, 12, 511-519.

34. B. Martín-Matute, E. Weis and M. J. Johansson, Chem. Eur. J., 2021, https://doi.org/10.1002/chem.202103510

35. E. Weis, M. A. Hayes, M. J. Johansson and B. Martín-Matute, iScience, 2021, 24.

36. E. Weis, M. J. Johansson and B. Martín-Matute, Chem. Eur. J., 2020, 26, 10185-10190.

37. E. Erbing, A. Sanz-Marco, A. Vázquez-Romero, J. Malmberg, M. J. Johansson, E. GómezBengoa and B. Martín-Matute, ACS Catal., 2018, 8, 920-925.

38. P. Amstutz, J. N. Pelletier, A. Guggisberg, L. Jermutus, S. Cesaro-Tadic, C. Zahnd and A. Plückthun, J. Am. Chem. Soc., 2002, 124, 9396-9403.

39. Y. Nishimura, H. Adachi, M. Kyo, S. Murakami, S. Hattori and K. Ajito, Bioorg. Med. Chem. Lett., 2005, 15, 2159-2162.

40. S. Schiesser, R. J. Cox and W. Czechtizky, Future Med. Chem., 2021, 13, 941-944.

41. T. Cernak, N. J. Gesmundo, K. Dykstra, Y. Yu, Z. Wu, Z.-C. Shi, P. Vachal, D. Sperbeck, S. He, B. A. Murphy, L. Sonatore, S. Williams, M. Madeira, A. Verras, M. Reiter, C. H. Lee, J. Cuff, E. C. Sherer, J. Kuethe, S. Goble, N. Perrotto, S. Pinto, D.-M. Shen, R. Nargund, J. Balkovec, R. J. DeVita and S. D. Dreher, J. Med. Chem., 2017, 60, 3594-3605.

42. E. Y. Wong and S. L. Diamond, Anal. Chem., 2009, 81, 509-514.

43. I. Sinclair, M. Bachman, D. Addison, M. Rohman, D. C. Murray, G. Davies, E. Mouchet, M. E. Tonge, R. G. Stearns, L. Ghislain, S. S. Datwani, L. Majlof, E. Hall, G. R. Jones, E. Hoyes, J. Olechno, R. N. Ellson, P. E. Barran, S. D. Pringle, M. R. Morris and J. Wingfield, Anal. Chem., 2019, 91, 3790-3794.

44. B. L. Tóth, A. Monory, O. Egyed, A. Domján, A. Bényei, B. Szathury, Z. Novák and A. Stirling, Chem. Sci., 2021, 12, 5152-5163. 